\title{
Utilisation des enrochements dans les ouvrages hydrauliques - Un nouveau guide technique européen
}

\author{
Céline Trmal ${ }^{1}$ et Sébastien Dupray ${ }^{2}$ \\ ${ }^{1}$ Ingénieur chargée d'études, CETMEF, Département Ports Maritimes \\ et Voies Navigables, \\ 2 Boulevard Gambetta - BP 60039 - 60321 Compiègne Cedex, \\ France \\ celine.trmal@equipement.gouv.fr \\ ${ }^{2}$ Chef de section, CETE de Lyon, Laboratoire Régional des Ponts et \\ Chaussées, \\ 25 avenue F. Mitterrand-BP 1 - 69674 Bron Cedex, France \\ sebastien.dupray@equipement.gouv.fr
}

\section{Résumé}

Le Manual on the use of rock in hydraulic engineering, communément appelé le Rock Manual a fait l'objet d'une mise à jour importante, menée par une équipe européenne (Royaume-Uni, Pays-Bas et France), de manière à intégrer les derniers travaux de recherches, le nouveau contexte normatif européen et des nouvelles considérations. Le projet d'actualisation arrive à son terme et se concrétise par la publication des versions anglaise et française. Cet article présente les motivations de la mise à jour, ainsi qu'une vue d'ensemble du contenu. Les changements principaux par rapport aux versions précédentes seront listés. Enfin, il sera montré comment l'utilisation du guide a été rendu plus facile.

\section{$\underline{\text { Abstract }}$}

The Manual on the use of rock in hydraulic engineering, commonly called the Rock Manual has been updated extensively, the update was led by an European team (United Kingdom, Netherlands and France) to include the latest research works, the new European standard context and the new concerns. The project comes now to its end with the publication of the English and French versions. This paper presents the motivations for the update, as well as the overview of the content. The essential changes from earlier versions are listed. Finally, it is shown how the user friendliness has been improved.

Mots-clés : Enrochement, digue, guide technique, ouvrage hydraulique, bonne pratique, Europe

Keywords : Armourstone, breakwater, guidance, hydraulic structures, good practice, Europe 


\section{Contexte}

L'enrochement naturel a toujours été un matériau très utilisé pour la construction des ouvrages hydrauliques de protection contre la houle et les courants de par sa grande disponibilité et son utilisation polyvalente. Dans les années 90, des efforts importants ont abouti à la production de plusieurs guides. Le guide technique Les enrochements ${ }^{8}$ publié par le Laboratoire Central des Ponts et Chaussées en collaboration avec le Centre d'Etudes Techniques Maritimes et Fluviales, se concentre sur l'aspect "matériaux". La première édition du Manual on the use of rock in coastal and shoreline engineering a été produite en 1991 résultant d'une collaboration entre le CIRIA (Construction Industry Research and Information Association, Royaume-Uni) et le CUR (Centre for Civil Engineering Research and Codes, Pays-Bas). Ce manuel s'intéresse à la conception, au dimensionnement et aux matériaux naturels dans le contexte de la construction d'ouvrages marins ou côtiers en enrochement. Une édition élargie au domaine des ouvrages en rivières et canaux ainsi qu'aux barrages en enrochement a été publiée par le CUR en 1995 sous le titre de Manual on the use of rock in hydraulic engineering 6 , plus connu sous le nom de Rock Manual. Les méthodes de construction y ont été aussi abordées.

D'autres publications sur les digues, endiguements et autres ouvrages en enrochements existent dont notamment le Shore Protection Manual ${ }^{9}$ du USACE (United States Army Corps of Engineers, Etats-Unis) mis à jour et réédité sous le nom du Coastal Engineering Manual ${ }^{10}$ en 2003.

\section{Motivations et approches de la nouvelle édition}

De nombreux changements dans le domaine des ouvrages en enrochement en site aquatique ont rendu la mise à jour du Rock Manual nécessaire, notamment :

- L'avancée capitale qu'a été la publication des normes européennes sur le matériau enrochement (EN $13383^{1,2}$ ) et la construction des ouvrages de génie civil (Eurocode ${ }^{3}$ ). Cela a rendu obsolète les éditions précédentes du Rock Manual. La mise en application de ces normes a paru être un moment clé pour mettre en place ce projet de mise à jour.

- Les importants progrès dans les recommandations et les pratiques pour la conception des ouvrages en enrochement.

- La prise de conscience accrue en matière de développement durable. Des recommandations de bonnes pratiques sont proposées. Les considérations de développement durable en matière de construction d'ouvrages en enrochement incluent la gestion et la meilleure utilisation des ressources et la réduction de l'impact des ouvrages sur l'environnement et si possible l'amélioration des zones d'habitat.

- Les retours d'expériences des utilisateurs et leurs commentaires sur l'utilisation des versions précédentes du Rock Manual qui ont directement influencé le contenu et l'organisation de la nouvelle édition. 
De plus, il était nécessaire de mettre à disposition des professionnels un document commun présentant les problématiques propres aux maîtres d'ouvrage et d'œuvre, aux bureaux d'ingénierie, aux producteurs et fournisseurs d'enrochement, aux entreprises, aux organismes de contrôle et aux laboratoires.

La nouvelle édition est le fruit d'une collaboration entre le Royaume-Uni, la France et les Pays-Bas. Elle a aussi bénéficié d'une relecture internationale de manière à assurer que ce guide donne des recommandations sur les pratiques mondiales en cours. Le manuel comprend aussi des résultats de recherches récentes, des informations techniques ainsi que des expériences pratiques acquises par les différents professionnels et décrit les bonnes pratiques actuelles. Un effort particulier a été fait pour indiquer les limitations actuelles dans la compréhension des phénomènes physiques complexes mis en jeu ainsi que la complémentarité des méthodes de conception, des formules empiriques et du jugement de l'ingénieur. Le manuel accentue la nécessité de prendre en compte la gestion de l'ouvrages tout au long de son cycle de vie. Les facteurs, autres que techniques qui doivent être pris en compte, par exemple les facteurs sociaux, environnementaux ou économiques sont aussi présentés.

\section{Contenu du manuel}

Le guide est autoporteur dans la mesure où il couvre la conception, le dimensionnement, la construction et la maintenance des ouvrages hydrauliques concernés ainsi que les matériaux de construction, i.e. les enrochements et les solutions alternatives proches à savoir les blocs artificiels en béton, les matériaux recyclés et les gabions.

Plus en détail, les chapitres s'articulent ainsi :

Chapitre 1 Introduction : présente l'objectif du guide, les aspects clés de sa mise à jour et l'organisation de son contenu

Chapitre 2 Considérations pour le projet et la conception des ouvrages en enrochement : donne les considérations essentielles pour un projet d'ouvrage en enrochement dans un environnement aquatique. Ce chapitre est un premier point de référence important car il propose un aide-mémoire des problèmes à résoudre. Le lecteur est encouragé à lire ce chapitre avant les autres. Le contenu est concis et ciblé pour en améliorer l'utilisation.

Chapitre 3 Matériaux : discute des différentes problématiques relatives à la sélection des matériaux naturels ou des alternatives présentées, incluant le gisement, la qualité et la durabilité, les propriétés et l'approvisionnement. Il décrit les différentes méthodes d'essai permettant de vérifier la propriété des enrochements et fait référence aux exigences européennes sur les enrochements $\left(\mathrm{EN} 13383^{1,2}\right)$. Ce chapitre est essentiel aux concepteurs qui veulent sélectionner des enrochements aux propriétés appropriées 
mais aussi aux concepteurs et aux producteurs-fournisseurs désireux d'en connaître plus sur les techniques de production.

Chapitre 4 Caractérisation du site et collecte des données : résume les méthodes de collecte de données et leur exploitation pour obtenir les conditions hydrauliques, géotechniques et liées à la glace. Ce chapitre est indispensable pour les concepteurs car il est le point de départ pour un dimensionnement, il est également important pour l'entreprise qui a besoin d'informations sur les conditions environnementales pour les opérations de construction.

Chapitre 5 Phénomènes physiques et outils de dimensionnement : présente les méthodes et les équations de dimensionnement en milieu marin et fluvial. Il couvre les performances hydrauliques, la stabilité et le comportement géotechnique. Les données d'entrée de ce chapitre sont les paramètres obtenus à partir des chapitres 3 et 4 .

Chapitre 6 Conception des ouvrages «à la mer»

Chapitre 7 Conception des barrages

Chapitre 8 Conception des ouvrages en rivière et canal : fournissent des recommandations opérationnelles pour la conception des différents types d'ouvrages. Ces chapitres s'appuient sur les chapitres 3, 4 et 5 et donnent des recommandations sur les dimensions et les détails des ouvrages, en prenant en compte les contraintes existantes, comme la constructibilité, les accès, la disponibilité des matériaux. Ces chapitres couvrent en particulier le tracé en plan, la géométrie des profils, les détails structurels (pied, crête, etc), les transitions et jonctions, l'influence de la construction sur le dimensionnement, les considérations de coût et de maintenance, la réparation et la réhabilitation d'ouvrages existants. La sélection des conditions de projet appropriées est aussi abordée dans ces chapitres.

Chapitre 9 Construction : traite (i) des équipements et du transport, (ii) des spécificités liées au site incluant la préparation du chantier, (iii) le contrôle qualité, incluant le placement et la densité de pose, (iv) le risque, l'hygiène et la sécurité sur chantier. Les méthodes de construction sont décrites pour les ouvrages hydrauliques les plus fréquents.

Chapitre 10 Surveillance, inspection, maintenance et réparation : donne des recommandations sur la maintenance et la gestion des ouvrages, l'évaluation des performances de l'ouvrage et les méthodes de réparation. 
Les principaux ouvrages hydrauliques traités sont les suivants :

\begin{tabular}{|c|c|c|c|}
\hline & Type d'ouvrage & Description & Fonctions \\
\hline \multirow{9}{*}{ 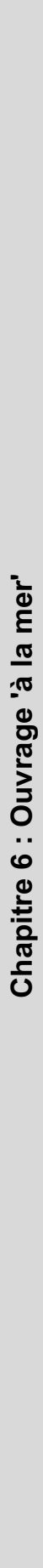 } & $\begin{array}{l}\text { Digue à talus, à } \\
\text { berme, mixte }\end{array}$ & $\begin{array}{l}\text { Généralement ouvrage } \\
\text { comprenant un talus, } \\
\text { composé d'un massif } \\
\text { d'enrochements de tailles } \\
\text { variables depuis les blocs } \\
\text { les plus lourds à } \\
\text { l'extérieur jusqu'aux } \\
\text { éléments les plus petits } \\
\text { formant le noyau }\end{array}$ & $\begin{array}{l}\text { - Abrite les navires et protège } \\
\text { les installations portuaires des } \\
\text { vagues et du courant ; } \\
\text { - Evite l'envasement des } \\
\text { chenaux d'accès. }\end{array}$ \\
\hline & $\begin{array}{l}\text { Protection en } \\
\text { enrochement des } \\
\text { ouvrages portuaires }\end{array}$ & $\begin{array}{l}\text { Généralement protection } \\
\text { en enrochement située au } \\
\text { pied des quais verticaux } \\
\text { ou sur les talus des quais } \\
\text { sur pieux. }\end{array}$ & $\begin{array}{l}\text { - Apporte une protection } \\
\text { contre les jets d'hélice et les } \\
\text { vagues induites par les } \\
\text { navires. }\end{array}$ \\
\hline & Revêtement & $\begin{array}{l}\text { Ouvrage de protection } \\
\text { normalement sur un talus } \\
\text { à protéger. }\end{array}$ & $\begin{array}{l}\text { - Protège le littoral contre } \\
\text { l'érosion; } \\
\text { - Limite le franchissement. }\end{array}$ \\
\hline & Protection de pied & $\begin{array}{l}\text { Berme en enrochement } \\
\text { construite en pied } \\
\text { d'ouvrage, où une érosion } \\
\text { est constatée }\end{array}$ & $\begin{array}{l}\text { - Prévient l'instabilité de la } \\
\text { défense côtière par érosion du } \\
\text { pied. }\end{array}$ \\
\hline & Epi & $\begin{array}{l}\text { Ouvrage en enrochement } \\
\text { généralement construit } \\
\text { perpendiculairement au } \\
\text { trait de côte }\end{array}$ & $\begin{array}{l}\text { - Intercepte le transport } \\
\text { sédimentaire longitudinal et } \\
\text { piège ainsi les sédiments. }\end{array}$ \\
\hline & Brise-lames & $\begin{array}{l}\text { Ouvrage en enrochement } \\
\text { généralement construit } \\
\text { parallèlement au rivage, } \\
\text { et non connecté à la côte }\end{array}$ & $\begin{array}{l}\text { - Intercepte et stabilise les } \\
\text { sédiments. }\end{array}$ \\
\hline & Digue submersible & $\begin{array}{l}\text { Ouvrage en enrochement } \\
\text { généralement construit } \\
\text { parallèlement à la côte en } \\
\text { pied de plage }\end{array}$ & $\begin{array}{l}\text { - Piège ou retient les } \\
\text { sédiments dans une position } \\
\text { élevée (soutient la plage). }\end{array}$ \\
\hline & $\begin{array}{l}\text { Protection en } \\
\text { enrochement des } \\
\text { conduites et des } \\
\text { câbles }\end{array}$ & $\begin{array}{l}\text { Couches en enrochement } \\
\text { ou remblaiement d'une } \\
\text { tranchée }\end{array}$ & $\begin{array}{l}\text { - Assure la stabilité et la } \\
\text { protection de la conduite ; } \\
\text { - Protège contre les impacts } \\
\text { par exemple des ancres ou } \\
\text { des engins de pêche. }\end{array}$ \\
\hline & $\begin{array}{l}\text { Protection en } \\
\text { enrochement contre } \\
\text { l'érosion des } \\
\text { structures offshore }\end{array}$ & $\begin{array}{l}\text { Protection en } \\
\text { enrochement autour de la } \\
\text { base ou en pied d'ouvrage }\end{array}$ & $\begin{array}{l}\text { - Prévient l'instabilité de } \\
\text { l'ouvrage par érosion du pied. }\end{array}$ \\
\hline
\end{tabular}




\begin{tabular}{|c|c|c|c|}
\hline & Type d'ouvrage & Description & Fonctions \\
\hline \multirow{4}{*}{ 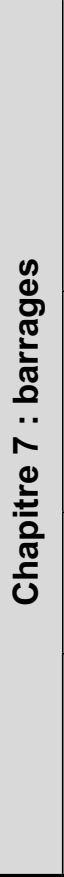 } & $\begin{array}{l}\text { Ouvrage de } \\
\text { fermeture de rivière } \\
\text { et d'estuaire }\end{array}$ & $\begin{array}{l}\text { Massif en enrochement, } \\
\text { quelquefois en tranche, le } \\
\text { plus souvent dimensionné } \\
\text { par les courants } \\
\text { importants lors de phase } \\
\text { finale de fermeture }\end{array}$ & $\begin{array}{l}\text { - Permet la dérivation de } \\
\text { rivières (temporaires ou } \\
\text { permanentes) par ex. pour } \\
\text { réguler la rivière, pour des } \\
\text { travaux, pour l'irrigation, pour la } \\
\text { navigabilité ; } \\
\text { - Ferme un estuaire. }\end{array}$ \\
\hline & $\begin{array}{l}\text { Barrage en } \\
\text { enrochement }\end{array}$ & $\begin{array}{l}\text { Ouvrage constitué } \\
\text { d'enrochement de } \\
\text { gradation très étendue, } \\
\text { souvent avec un noyau } \\
\text { étanche }\end{array}$ & - Retient l'eau. \\
\hline & $\begin{array}{l}\text { Protection des } \\
\text { barrages réservoir }\end{array}$ & $\begin{array}{l}\text { Protection des parements } \\
\text { et déversoir de barrages } \\
\text { réservoir }\end{array}$ & - Protège le noyau de l'érosion. \\
\hline & $\begin{array}{l}\text { Ouvrage de } \\
\text { régulation (seuils, } \\
\text { déversoirs, } \\
\text { barrages...) }\end{array}$ & $\begin{array}{l}\text { Ouvrage bas par rapport } \\
\text { au niveau d'eau } \\
\text { dimensionné pour les } \\
\text { écoulements internes et } \\
\text { les débordements }\end{array}$ & $\begin{array}{l}\text { - Protège les zones avales de } \\
\text { ces ouvrages. }\end{array}$ \\
\hline \multirow{6}{*}{ 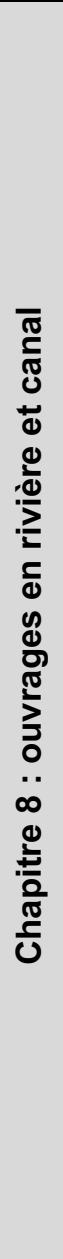 } & Protection de berges & $\begin{array}{l}\text { Revêtement en } \\
\text { enrochements ou en } \\
\text { gabions mis en place sur } \\
\text { la berge }\end{array}$ & $\begin{array}{l}\text { - Protège les berges contre } \\
\text { l'érosion; } \\
\text { - Contrôle le développement de } \\
\text { la rivière en empêchant } \\
\text { l'attaque des ouvrages } \\
\text { adjacents. }\end{array}$ \\
\hline & Protection de lit & $\begin{array}{l}\text { Couches d'enrochement } \\
\text { ou de gabion mis en place } \\
\text { sur le lit d'une rivière }\end{array}$ & $\begin{array}{l}\text { - Prévient l'érosion du lit, } \\
\text { incluant les affouillements dus } \\
\text { au batillage. }\end{array}$ \\
\hline & Épi & $\begin{array}{l}\text { Ouvrage perpendiculaire à } \\
\text { la berge protégé par des } \\
\text { enrochements }\end{array}$ & $\begin{array}{l}\text { - Contrôle le tracé de la rivière ; } \\
\text { - Réduit la section pour assurer } \\
\text { un niveau d'eau suffisant ; } \\
\text { - Éloigne les courants des } \\
\text { berges. }\end{array}$ \\
\hline & $\begin{array}{l}\text { Digue et } \\
\text { endiguement }\end{array}$ & $\begin{array}{l}\text { Ouvrage longitudinal } \\
\text { protégé par des } \\
\text { enrochements }\end{array}$ & $\begin{array}{l}\text { - Prévient l'érosion des berges ; } \\
\text { - Contrôle les courants et le } \\
\text { tracé de la rivière, en } \\
\text { empêchant la submersion et } \\
\text { l'érosion des ouvrages } \\
\text { adjacents. }\end{array}$ \\
\hline & $\begin{array}{l}\text { Ouvrages spéciaux: } \\
\text { Passe à poissons }\end{array}$ & $\begin{array}{l}\text { Ouvrage barrant } \\
\text { partiellement la rivière, } \\
\text { utilisant quelque fois des } \\
\text { enrochements }\end{array}$ & $\begin{array}{l}\text { - Permet aux poissons de } \\
\text { franchir un obstacle en assurant } \\
\text { un niveau d'eau ou un débit } \\
\text { approprié ; }\end{array}$ \\
\hline & $\begin{array}{l}\text { Protection de piles de } \\
\text { pont }\end{array}$ & $\begin{array}{l}\text { Berme ou protection de } \\
\text { fond en enrochement } \\
\text { autour des piles de ponts }\end{array}$ & $\begin{array}{l}\text { - Prévient l'érosion du lit de la } \\
\text { rivière autour des piles de } \\
\text { ponts. }\end{array}$ \\
\hline
\end{tabular}




\section{Changements par rapport aux versions précédentes}

La version réactualisée du Rock Manual contient les nouveaux aspects suivants :

- des recommandations pour la conception et la construction utilisant des matériaux alternatifs aux enrochements naturels, ie gabions et blocs préfabriqués en béton ;

- des spécifications réactualisées pour la construction des ouvrages en enrochement ;

- des références croisées vers les nouvelles normes européennes des enrochements (EN $13383^{1,2}$ );

- les nouvelles recherches sur l'intégrité des enrochements, sur le placement et la densité de pose des enrochements ;

- les nouvelles avancées en matière de prédiction de la production des carrières et les distributions granulométriques des enrochements ;

- une réactualisation des recommandations sur le franchissement des ouvrages par la houle et sur la transmission de la houle ;

- une réactualisation des recommandations sur la distribution des hauteurs de houle en eaux peu profondes ;

- des recommandations réactualisées sur le dimensionnement géotechnique en faisant référence aux Eurocodes ${ }^{3}$;

- des nouvelles recommandations pour le calcul des forces de la houle sur le mur de couronnement ;

- des nouvelles recommandations sur la stabilité du talus arrière des ouvrages en enrochement ;

- des nouvelles recommandations sur la stabilité des massifs d'enrochements situés près du fond ;

- des recommandations réactualisées sur la stabilité des carapaces en eaux peu profondes ;

- des nouvelles recommandations pour la conception et la construction des digues à berme stable statiquement ;

- deux nouvelles sections sur le comportement des ouvrages soumis à l'action de la glace ;

- une nouvelle section sur la conception des protections en enrochements des ouvrages portuaires ;

- un chapitre complètement révisé sur la maintenance et la gestion des ouvrages.

Par rapport aux versions précédentes les sujets suivants ne sont plus traités :

- les plages en galets, car ce point est couvert dans d'autres manuels sur la conception des plages (par ex. The beach management manual ${ }^{4}$ );

- les recommandations détaillées sur l'érosion et les affouillements car ce sujet est bien couvert dans d'autres ouvrages (par ex. Scour manual ${ }^{7}$ );

- les annexes sur la collecte des données hydrauliques et géotechniques. 


\section{Amélioration de l'utilisation du guide}

Un effort particulier a été mené par l'équipe du projet pour améliorer la lisibilité et l'utilisation du guide. Des logigrammes présents en début de chaque chapitre permettent aux lecteurs de retrouver l'information recherchée plus facilement. L'interaction entre les différents chapitres a été accentuée par la présence importante des références croisées.

\section{Exemple de logigramme :}

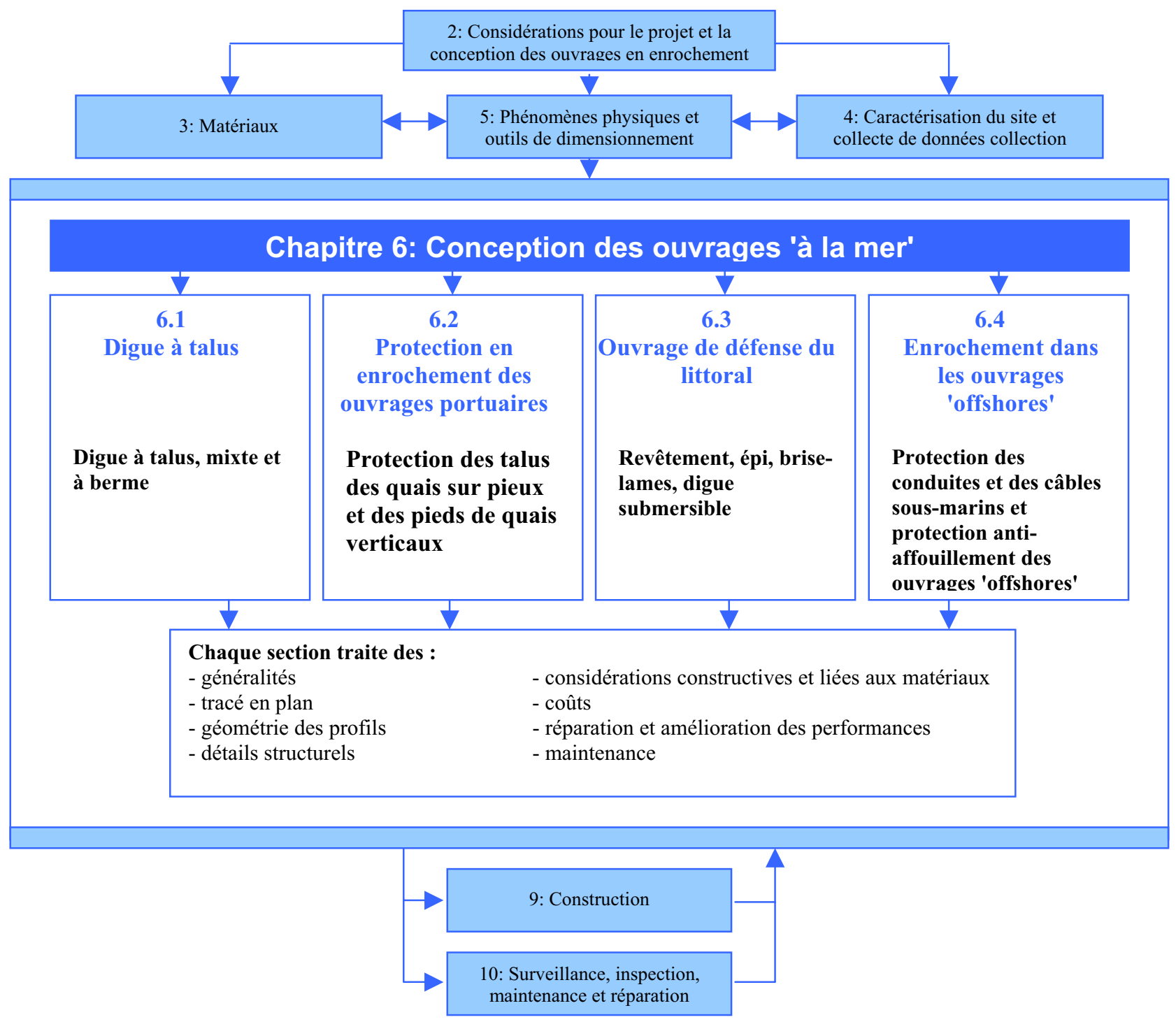

\section{Conclusion}

Dans cet article, les motivations et l'essentiel du contenu du Rock Manual ont été présentés. Le guide sera publié par le CIRIA en anglais en fin d'année 2006 et en français début 2007. En résumé :

- Le nouveau Rock Manual est un guide technique présentant un bilan des bonnes pratiques dans l'utilisation des enrochements dans les ouvrages 
hydrauliques. Des innovations sont mentionnées pour tenir le lecteur au fait des derniers développements dans les domaines scientifiques et techniques concernés. Le guide technique propose des méthodes et des outils appropriés à la résolution de problèmes identifiés mais il n'a légalement le statut ni de norme ni de recommandation.

- Les lecteurs ciblés pour ce guide sont les maîtres d'ouvrage et d'œuvre, les bureaux d'ingénierie, les producteurs et fournisseurs d'enrochement, les entreprises, les organismes de contrôle et les laboratoires.

- Les structures visées par ce guide sont les ouvrages en enrochement, notamment les ouvrages maritimes, côtiers et portuaires, les ouvrages en rivières et en voies navigables, les barrages en enrochement et les protections de barrages.

- Le guide est autoporteur. Ainsi, il couvre la conception, le dimensionnement, la construction et la maintenance des ouvrages concernés, ainsi que les matériaux de construction.

- Le guide sera disponible en anglais et en français au format papier et électronique.

Ce projet est un exemple de collaboration entre des acteurs publics et privés au sein de l'Europe pour aboutir à un document innovant et pratique.

\section{$\underline{\text { Remerciement }}$}

Le guide a été mis à jour par une équipe de projet anglaise, française et hollandaise représentée respectivement par le CIRIA, CETMEF et le CUR. Les principaux partenaires sont, au Royaume-Uni, HR Wallingford, Imperial College, Halcrow et l'Université de Southampton ; au Pays-Bas, Rijkswaterstaat, Delft Hydraulics, TU Delf, Royal Boskalis Westminster ; et en France, CETMEF, CETE de Lyon, SOGREAH, EDF, LCPC.

\section{$\underline{\text { Références }}$}

1 AFNOR (2003), NF EN 13383-1 Enrochements - Partie 1 : Spécifications Indice de classement : P18-661-1, $45 \mathrm{p}$.

2 AFNOR (2003), NF EN 13383-2 Enrochements - Partie 2 : Méthodes d'essai, Indice de classement : P18-661-2, $44 \mathrm{p}$.

3 AFNOR (2005), NF EN 1997-1 Eurocode 7 : calcul géotechnique - Partie 1 : règles générales, Indice de classement : P94-251-1, 145 p.

4 CIRIA (1996), The Beach Management Manual, R153, CIRIA, 448 p.

5 CIRIA/CUR (1991), Manual on the use of rock in coastal and shoreline engineering. CIRIA special publication 83 , CIRIA, 607p.

6 CUR/RWS (1995), Manual on the use of rock in hydraulic engineering. Report 169, AA Balkema, 800p.

7 Hoffmans, G J C M and Verheij, H J (1997), Scour Manual, Balkema, Rotterdam. 
Session 6 - Ouvrages et aménagements portuaires

8 LCPC (1989), Les enrochements. Ministères de l'Equipement, LCPC, 106p.

9 USACE (1984), Shore protection manual, $4^{\text {th }}$ edition

10 USACE (2003), Coastal Engineering Manual (disponible sur le site : http://chl.erdc.usace.army.mil) 